\title{
The Role of Inertial Sensitivity in Motor Planning
}

\author{
Philip N. Sabes, ${ }^{1}$ Michael I. Jordan, ${ }^{1}$ and Daniel M. Wolpert ${ }^{2}$ \\ ${ }^{1}$ Department of Brain and Cognitive Sciences, Massachusetts Institute of Technology, Cambridge, Massachusetts 02139, \\ and ${ }^{2}$ Sobell Department of Neurophysiology, Institute of Neurology, London WC1N 3BG, United Kingdom
}

To achieve a given motor task a single trajectory must be chosen from the infinite set of possibilities consistent with the task. To investigate such motor planning in a natural environment, we examined the kinematics of reaching movements made around a visual obstacle in three-dimensional space. Within each session, the start and end points of the movement were uniformly varied around the obstacle. However, the distribution of the near points, where the paths came closest to the obstacle, showed a strong anisotropy, clustering at the poles of a preferred axis through the center of the obstacle. The preferred axes for movements made with the left and right arms were mirror symmetric about the midsagittal plane, suggesting that the anisotropy stems from intrinsic properties of the arm rather than extrinsic visual factors. One account of these results

Movement planning can be considered as the specification of a movement trajectory from the infinite number of possible trajectories that are consistent with a given task (for review, see Wolpert, 1997). Although many theories of motor planning have been proposed, they fall into two main classes: extrinsic and intrinsic. Proponents of extrinsic planning suggest that the process is hierarchical, beginning with the specification of the trajectory of the end point, such as the hand or finger, in extrinsic visual space (Bernstein, 1967; Morasso, 1981; Abend et al., 1982; Flash and Hogan, 1985; Flash and Gurevich, 1991; Lackner and DiZio, 1994; Shadmehr and Mussa-Ivaldi, 1994; Flanagan and Rao, 1995; Wolpert et al., 1995; Sabes, 1996). This class of models suggests that the end point trajectory of visually guided tasks will depend only on the visual task constraints. Proponents of intrinsic planning suggest that the intrinsic kinematics of the trajectory (e.g., the trajectory of joint configurations) are planned directly, taking into account intrinsic properties of the limb (Soechting and Lacquaniti, 1981; Kaminsky and Gentile, 1986; Soechting and Flanders, 1989; Uno et al., 1989; Flanagan and Ostry, 1990; Desmurget et al., 1995). Theoretical models of both classes of motor planning have been based mainly on the optimal control framework in which planning is considered as the process of finding the trajectory, which minimizes some cost associated with the movement. However, within that framework cost functions that depend on both purely intrinsic (Uno et al., 1989) or extrinsic

Received Dec. 17, 1997; revised May 8, 1998; accepted May 13, 1998.

This project was supported by grants from the Wellcome Trust and the US Office of Naval Research. P.N.S. was supported by a training grant from the National Institute of General Medical Sciences. We thank N. Hogan for many helpful discussions.

Correspondence should be addressed to Philip N. Sabes, Division of Biology, 216-76, California Institute of Technology, Pasadena, CA 91125.

Copyright (C) 1998 Society for Neuroscience $\quad 0270-6474 / 98 / 185948-10 \$ 05.00 / 0$ is a sensitivity model of motor planning, in which the movement path is skewed so that when the hand passes closest to the obstacle, the arm is in a configuration that is least sensitive to perturbations that might cause collision. To test this idea, we measured the mobility ellipse of the arm. The mobility minor axis represents the direction in which the hand is most inertially stable to a force perturbation. In agreement with the sensitivity model, the mobility minor axis was not significantly different from the preferred near point axis. The results suggest that the sensitivity of the arm to perturbations, as determined by its inertial stability, is taken into account in the planning process.

Key words: human psychophysics; visuomotor control; motor planning; reaching; obstacle avoidance; optimal control; theoretical model

(Flash and Hogan, 1985) parameters can account fairly well for simple point-to-point reaching data.

Part of the difficulty in resolving the extrinsic-intrinsic controversy lies in the lack of rich task constraints in the extensively studied experimental paradigm of point-to-point reaching. In an attempt to move toward task constraints such as those present in everyday goal directed movement, Sabes and Jordan (1997) investigated reaching in the presence of an obstacle. By considering movements that were identical except for the rotation of the start and end points around the tip of the obstacle, they found a systematic variation in the path, suggesting that movements are not planned based purely on the extrinsic task specification. These variations support a model in which planning takes account of the sensitivity of the arm to external perturbations or uncertainty in joint level control or proprioception. The model posits that paths are chosen to minimize the sensitivity of the arm to perturbations in the direction of the obstacle when the arm is at the point of nearest approach to the obstacle.

Here, we present an obstacle avoidance experiment in three dimensions. As in the previous work by Sabes and Jordan (1997), the task is devised so that the task constraints have a rotational symmetry across trials, but here we explore three different axes of symmetry. This paper addresses a number of unresolved issues.

First, we investigate whether the path variations observed in obstacle avoidance movements can be explained in terms of a perceptual, rather than motor, anisotropy. We conducted two identical sets of experiments with each participant, once with each arm. If the path asymmetry is attributable to intrinsic properties of the arm, then the paths from the two arms should be mirror symmetric about the midsagittal plane. However, if the asymmetry is attributable to extrinsic factors, such as a visual perceptual process, the asymmetry should be independent of the arm used. 


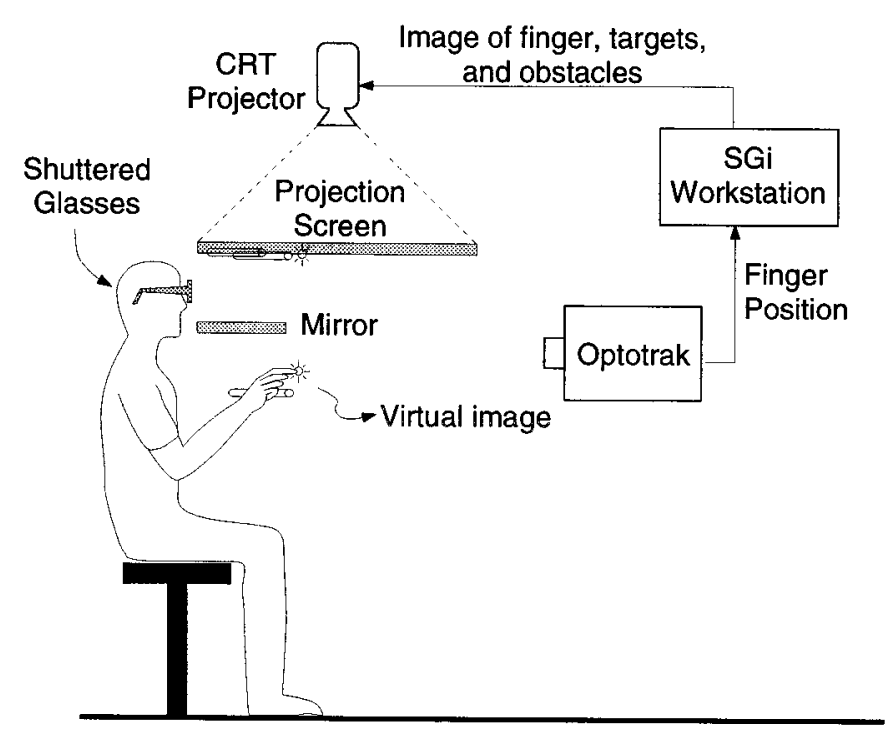

Figure 1. Three-dimensional virtual visual feedback system.

Second, the results of Sabes and Jordan (1997) suggest that the inertial properties of the arm could play a central role in the process of planning obstacle avoidance movements, but measurements of the arm's inertia were not available. Here, we make direct measurements of the inertia and compare the predictions of the sensitivity model to the data from the obstacle rotation experiment.

Finally, the majority of motor-planning studies have been restricted to movements involving an interaction with a planar surface or $2 \mathrm{df}$ manipulandum. This method places these movements into the domain of compliant control (Hollerbach, 1982), in which an entirely different strategy may be used for planning movements (Desmurget et al., 1997). This is problematic when trying to investigate the way that the CNS interacts with a particular object, namely the obstacle, because additional constraints could confound our results. In the present study, participants' arms were completely unconstrained, and movements were made around obstacles in a variety of orientations.

\section{MATERIALS AND METHODS}

Five participants, two left-handed and three right-handed, gave their informed consent and took part in the experiment. All participants had normal or corrected to normal vision. Two authors (P.S. and D.W.) were participants, and the remaining three participants were naive as to the purpose of the study.

\section{Obstacle rotation}

Apparatus. A three-dimensional virtual visual feedback system (Fig. 1) was used to record the motion of the hand and to generate the obstacles and feedback of the hand's position. An Optotrak 3020 infrared position monitoring system (Northern Digital, Waterloo, Ontario, Canada) tracked the position of an infrared emitting diode (IRED) mounted on the tip of the participant's index finger. These positions were sampled by a Silicon Graphics (Mountain View, CA) Indigo (SGi) 2 XZ workstation at $200 \mathrm{~Hz}$ and used both on-line to drive the visual display as well as stored for spatial analysis of the paths.

The targets and feedback of finger position were presented as virtual three-dimensional images. This was achieved using a cathode ray tube projector (Electrohome, Rancho Cucamonga, CA; Marquee 8000 with a P43 low-persistence phosphor green tube) driven by the SGi workstation to project an image onto a horizontal rear projection screen suspended above the participant's head. A horizontal front-reflecting semisilvered mirror was placed face up below the participant's chin $(30 \mathrm{~cm}$ below the projection screen). The participant viewed the reflected image of the rear

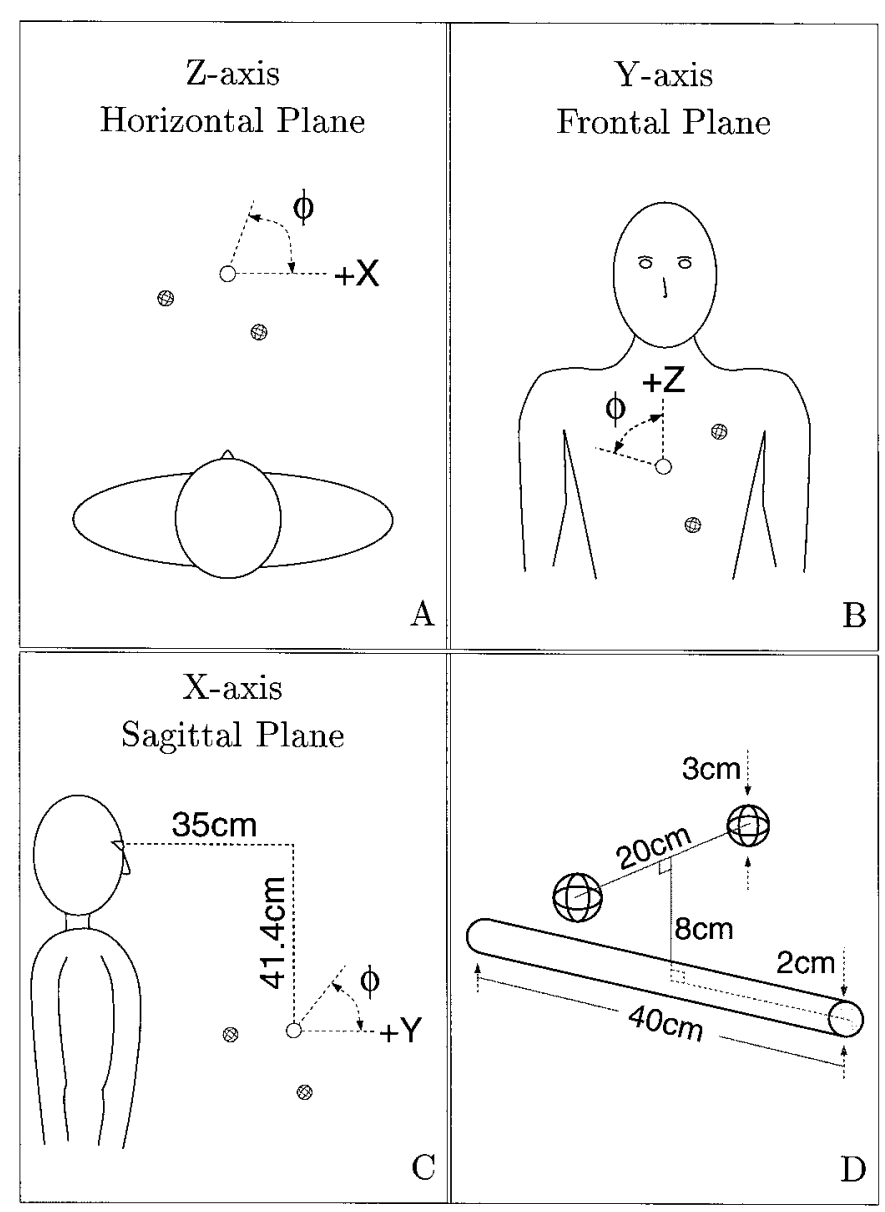

Figure 2. Visual scene for the obstacle rotation experiment. $A-C$, Visual scene at sample presentation angles for each of the three planes of movement. The viewer is looking down the length of the obstacle (open circle). Above each figure, the obstacle axis and the plane of rotation are listed. $D$, Obstacle and start and target spheres, with dimensions labeled.

projection screen through field-sequential shuttered glasses (Crystal Eyes; Stereo-graphic Inc.) by looking down at the mirror. The SGi workstation displayed left and right eye images $(1280 \times 500$ pixels $)$ of the scene to be viewed at $120 \mathrm{~Hz}$. The shuttered glasses alternately blanked the view from each eye in synchrony with the display, allowing each eye to be presented with the appropriate planar view. Participants therefore perceived a three-dimensional scene. A coordinate frame was chosen for the workspace with the $X$-axis lying along the transverse direction, the $Y$-axis along the sagittal direction, the $Z$-axis along the vertical, and an origin located at the center of the eyes (Fig. $2 A-C$ ). The workspace for the virtual feedback was centered at $(0.0,35.0,-41.4) \mathrm{cm}$, which will be referred to as the center point.

Before each experiment, the visual feedback system was calibrated to ensure that the absolute positions determined by the Optotrak were in register with the perceived three-dimensional location of the visual feedback. By illuminating the semisilvered mirror from below, the virtual image and the IRED could be lined up by eye. Each participant calibrated on 30 target locations uniformly distributed throughout the workspace. A linear regression fit of image position to IRED position was performed, and the results were then used on-line to position the targets and hand feedback images. Finally, participants were asked to point to 10 more targets to validate the regression fit. Only participants who achieved a validation root mean square error of $<0.8 \mathrm{~cm}$ were used in the experiment.

During the experiment an opaque sheet was fixed beneath the semisilvered mirror to block a direct view of the arm, and the room was darkened. Hand feedback was then provided by a $1 \mathrm{~cm}$ white wire cube in the virtual scene. The targets were presented as 3-cm-diameter spheres and the obstacle as a 2-cm-diameter cylinder. 


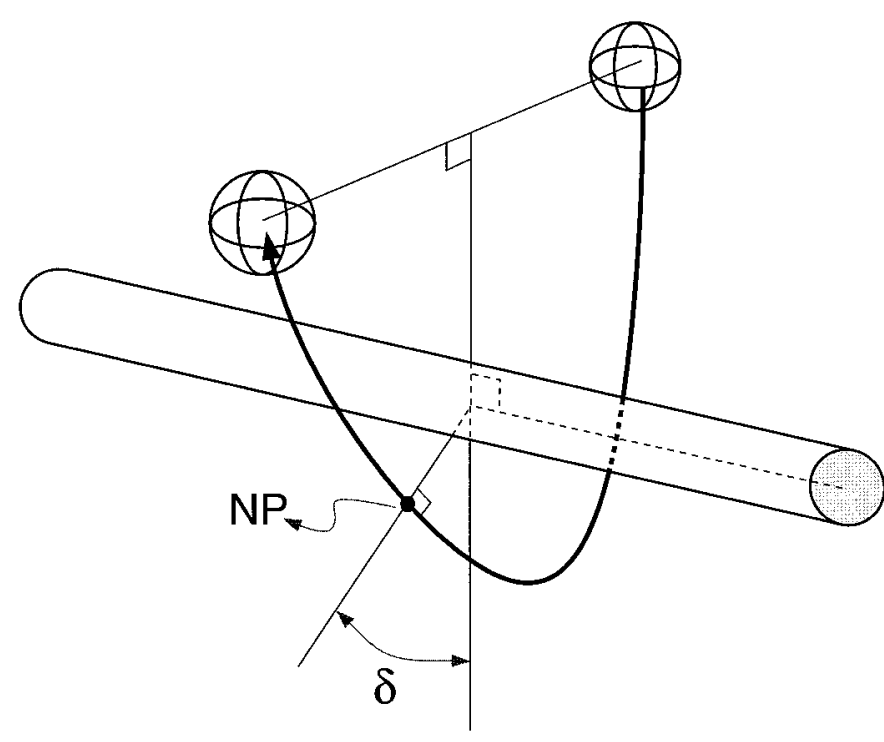

Figure 3. Definitions of the path near point $(N P)$ and the near point angle, $\delta$.

Procedure. Each trial began with a white (start) sphere, a blue (target) sphere, and a red cylindrical obstacle appearing in the workspace (Fig. $2 D$ ). Participants were instructed to move their finger into the start sphere and wait for a tone, at which point they were to reach around the obstacle to the target sphere, making sure to avoid hitting the obstacle with their finger (a sample path is shown in Fig. 3). If the fingertip collided with the obstacle, or if participants attempted to go directly to the target sphere without going around the obstacle, a low tone was sounded, and the trial was restarted. Otherwise, when the participant's fingertip came to rest in the target sphere, a high tone sounded, and the screen went blank until the next trial. Participants were given no further instructions, except to move naturally and comfortably.

The experiment was divided into blocks during which the cylindrical obstacle was fixed in space with its center at the center point of the workspace and its length lying along either the $X$-, $Y$-, or $Z$-axis. Within a block, the start and target points were always located in the plane that passed through the center point, perpendicular to the obstacle. The presentation angle $\phi$ determined the orientation of the start and target points relative to the obstacle. Therefore, within each block the geometry of the start and target spheres and the cylindrical obstacle were, apart from a single rotation, identical. $\phi$ is defined as the angle of the line passing from the intertarget axis through the obstacle, as shown in Figure $2 A-C$.

Trials occurred in "there-and-back" pairs: identities of the start and target circles were switched within a pair, but the presentation angle was held fixed. A trial block consisted of 120 pseudo-randomly ordered movement pairs with presentation angles located at $3^{\circ}$ increments around the circle. Before each block, participants were given $\sim 10$ practice trials to familiarize them with the upcoming task. Participants performed two sessions on different days, one with the right hand and one with the left. Each session consisted of three blocks, one with the obstacle along each axis. The order of the sessions and blocks within a session were randomized.

Data analysis. We define the path near point as the locus on the path that comes closest to the obstacle. We also define a near point angle, $\delta$, as the angle of the line connecting the obstacle and the near point measured with respect to the perpendicular bisector defining the presentation angle (Fig. 3). Because the end point (i.e., fingertip) paths lay primarily in the plane perpendicular to the obstacle, and motion along the obstacle does not change the distance to the obstacle, the near point angle was defined in the plane perpendicular to the obstacle.

\section{Mobility measurements}

We will consider a sensitivity model for path planning that relies on the notion of the arm's mobility. The mobility matrix is the inverse of the joint inertia matrix transformed into Cartesian space (Hogan, 1985). Formally, it is defined as:

$$
W(\theta)=J(\theta) I^{-1}(\theta) J^{\prime}(\theta),
$$

where $I(\theta)$ is the inertia matrix of the arm, and $J(\theta)$ is the Jacobian of the arm, both of which are functions of the joint configuration of the arm, $\theta$. The prime denotes the matrix transpose. The mobility relates a perturbative force, $\mathbf{f}$, at the end point to the resulting acceleration:

$$
\mathbf{a}=W \mathbf{f} .
$$

We measured the mobility of the left arm and the right arm at the center point for four of the five participants in the obstacle rotation experiment (including one author, D.W.). By repeatedly perturbing the hand, held stationary at the center point, with forces in variety of directions, we were able to estimate the mobility matrix by measuring the resulting hand acceleration and using the relationship of Equation 2.

Apparatus. The experimental apparatus used for the mobility measurement was the same as in the obstacle rotation experiment, except that participants grasped the handle of a lightweight, carbon fiber robotic manipulator (Phantom haptic interface; Sensable Devices, Cambridge, $\mathrm{MA}$ ). This robot, which is free to move in three dimensions, can exert forces of up to $20 \mathrm{~N}$ in any direction in three-dimensional space (backdrive friction, $0.02 \mathrm{~N}$; closed loop stiffness, $1 \mathrm{~N} / \mathrm{mm}$; apparent mass at the tip, $<150 \mathrm{gm}$ ). A custom-designed handle allowed rotation about the center in all three directions so that no torques would be transferred to the hand.

Procedure. At the beginning of each measurement, a target circle appeared in the visual display at the center of the workspace, and the manipulandum assisted the participant back to this position by simulating a weak spring attached to that point. When the participant was within 2 $\mathrm{cm}$ of the center point and the hand velocity was $<1 \mathrm{~cm} / \mathrm{sec}$ for at least $200 \mathrm{msec}$, the robot produced an $8.0 \mathrm{~N}$ force pulse of $200 \mathrm{msec}$ duration in a specified direction. The position of the participant's hand was monitored with the Optotrak at $1500 \mathrm{~Hz}$ for the duration of the pulse.

An experiment consisted of 72 pseudo-randomly ordered force perturbations at $5^{\circ}$ intervals around the circle in either the sagittal $(X)$, frontal $(Y)$, or horizontal $(Z)$ planes (capital letters refer to the cardinal axis, which is perpendicular to the respective plane). For each participant, six experiments were conducted, one for each arm in each of the three planes.

Data analysis. For a constant perturbative force, Equation 2 predicts a constant acceleration. Thus, ignoring for the moment the effects of the nonlinear terms of the dynamics and participants' reactions to the perturbation, we expect the hand position to be a quadratic function of time:

$$
\left[\begin{array}{l}
x_{t} \\
y_{t}
\end{array}\right]=\frac{\left[\begin{array}{lll}
x_{0} & v_{x_{0}} & a_{x_{0}} / 2 \\
y_{0} & v_{y_{0}} & a_{y_{0}} / 2
\end{array}\right]}{A}\left[\begin{array}{c}
1 \\
t \\
t^{2}
\end{array}\right] .
$$

The matrix of parameters $A$ was estimated with linear regression over varying temporal windows for each trial. For this analysis, the time origin was chosen as the midpoint of the interval, meaning that twice the third column of the matrix $A$ is an estimate of the acceleration at the center of the temporal window under consideration. We also calculated the $R^{2}$ statistic of each regression: the proportion of variance in position accounted for by the regression.

The mobility matrix was estimated by regressing the acceleration measurements from a particular trial on the direction of the perturbative forces, according to the linear relationship of Equation 2. Because we are only concerned with the shape of the mobility matrix, not its absolute size, the forces (and thus the resulting W estimates) were arbitrarily scaled. Because we computed mobility estimates within a single experiment, the resulting quantities are the $2 \times 2$ mobility matrices for each of the three cardinal planes.

Finally, the mobility, as defined in Equation 1, is necessarily symmetric. However, the estimation procedure described above does not constrain our estimates of $W$ to be symmetric; they may contain an antisymmetric component known as curl. There are a variety of factors that could contribute to the curl in our mobility estimates. First, the marker was not positioned exactly at the point where the force acted on the hand, so there may be some rotation in the data. Second, noise in the acceleration measurements will result in spurious nonsymmetric components to the least squares estimate of $W$. In particular, the actual forces delivered to the hand were not ideal force pulses but contained some non-negligible temporal dynamics. We expect this factor to be worse with shorter time windows. Third, Equation 2 only relates perturbative forces to the acceleration that directly results from the perturbation and only at the location for which $W$ is computed. The longer the window we use, the 
further the arm will be from the center point, and the greater the hand's velocity will be, meaning that other terms of the dynamics will play a larger role in the arm's acceleration. Also, at longer intervals beyond the stimulus onset, participants' reactions to the perturbation will be a factor.

Our theoretical interpretation of the mobility requires the existence of real eigenvectors for $W$, but a curl component in our mobility estimate can lead to complex eigenvalues. We therefore used a symmetrized version of our estimate $W$ for further analysis: $W_{\mathrm{s}}=\left(W+W^{\prime}\right) / 2$. As a measure of the curl in our mobility estimate, we will consider the ratio of the absolute value of the determinants of the antisymmetric and symmetric components of $W,\left|\operatorname{det}\left(W-W^{\prime}\right)\right| /\left|\operatorname{det}\left(W_{\mathrm{s}}\right)\right|$, which we will call the curl index. Note that the index is $<1$ when the mobility estimate's symmetric part is larger than its antisymmetric part.

\section{Sensitivity model}

Sabes and Jordan (1997) showed that in a planar, 2 df version of the obstacle rotation experiment, trajectory near points tended to cluster at opposite poles of the center point, on an axis approximately aligned with the orientation of the forearm. To account for this effect, they proposed a sensitivity model for the planning of obstacle avoidance movements. We will briefly review the model here.

In the obstacle avoidance task, the only constraint on the movement, other than the start and target points, is to avoid collision with the obstacle. Thus, it would be desirable to choose a path that minimizes the sensitivity of the arm to sensor or actuator uncertainty or external perturbations in the direction of the obstacle. The sensitivity model suggests that the near point of a path should be chosen to lie close to the axis of minimum sensitivity to perturbation or noise. This axis is called the (near point) preferred axis. Sabes and Jordan (1997) introduced three definitions of sensitivity - one purely kinematic, one based on the arm's elastic properties, and one based on its inertial properties-the last of which was shown to best account for the data from the planar obstacle rotation experiment. Here, we concentrate on that last measure, the arm's mobility $W$ (see Methods, Mobility measurements).

The eigenvectors of $W$ have a simple interpretation: the major (minor) eigenvector is the direction along which force perturbations have the largest (smallest) effect. Thus, the sensitivity model would predict that the near points should cluster toward the mobility minor axis. In other words, the mobility minor axis should be the near point preferred axis. We tested this theory by comparing it with the results from the two experiments presented in this paper.

Data analysis. To assess whether the near points cluster about a preferred axis, we examined the near point angle, $\delta$, as a function of presentation angle $\phi$. Consider the case in which movement paths are perfectly symmetric (i.e., swapping target and start positions does not change the path), and the apex of the movement comes closest to the obstacle. Here, all near points would lie along the axis defined by the presentation angle, and $\delta$, which is the difference angle between the near point and the presentation angle, would always be 0 , independently of $\phi$. The sensitivity model suggests that the near point will lie not at $\delta=0$ but, rather, some portion of the way from there to a preferred axis. This prediction can be formalized into a statistical model of the dependence of $\delta$ on $\phi$ :

$$
\delta=b(\omega-\phi) \% 180+\epsilon,
$$

where $\epsilon$ is zero mean, normally distributed noise with $\mathrm{SD} \sigma_{\epsilon}$, the "signed modulus," $y=x \% 180$, is defined as the $y$ in the interval $(-90,90)$ such that $x=y+180 n$ for some integer $n$, and all angles are in degrees. The two parameters of the model are the preferred axis $\omega$ and the slope $b$. The latter is a measure of the strength of the dependence of $\delta$ on $\phi$. This model describes a piecewise linear relationship between $\delta$ and $\phi$, in which the preferred axis $\omega$ acts as an attractor. Given a data set we can find the maximum likelihood parameter values, the values that best account for the experimental data, as well as confidence limits on those estimates. The details of this calculation are given in a previous article by Sabes and Jordan (1997). The estimated confidence interval for the preferred axis is not necessarily symmetric about the maximum likelihood value. It is also important to note that the slope $b$ plays the same role here as in standard linear regression: if $b$ is significantly different from zero, the null hypothesis that $\delta$ does not depend on $\phi$ (i.e., a strictly intrinsic planning model) is rejected in favor of the preferred axis model.

We will also have occasion to ask whether two sets of axial data (e.g., near point preferred axes and mobility minor axes) have the same mean. Because the data lie on a circular domain, we cannot use standard linear
Presentation Angles Near 90
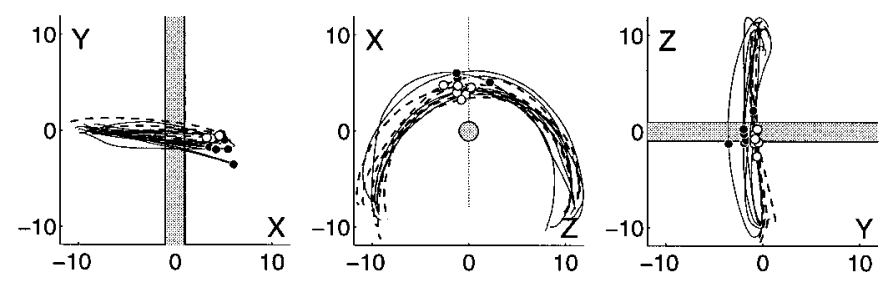

Presentation Angles Near $135^{\circ}$
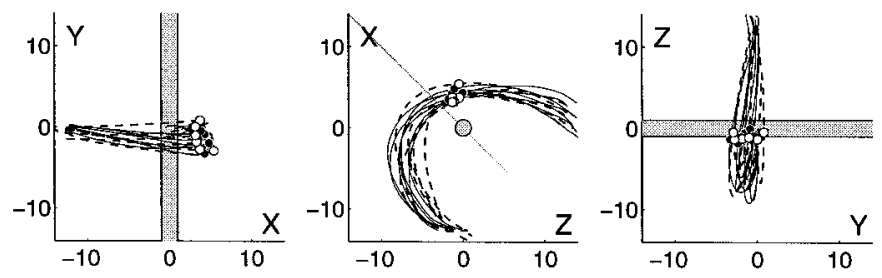

Presentation Angles Near $180^{\circ}$
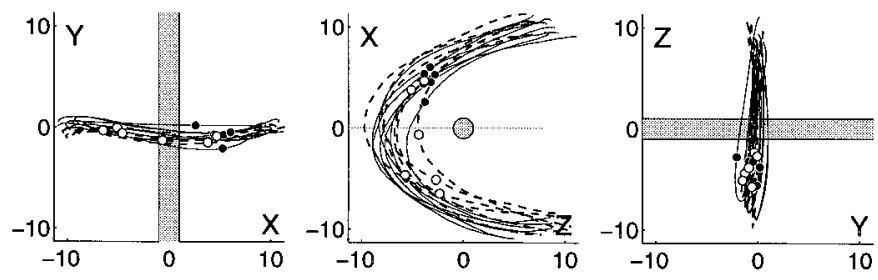

Figure 4. Sample obstacle avoidance paths. All three rows show sample plots from the same trial block: participant P.S., $Y$-axis, left hand. The three plots of each row present the same paths projected onto the three cardinal axes. Each row displays all trials with presentation angles within $10^{\circ}$ of the value in the title. Solid lines are for counterclockwise movements; dashed lines are for clockwise. The black and gray circles mark the counterclockwise and clockwise near points, respectively. The line $\delta=0$ is shown by the dotted line bisecting the obstacle. Note that in these plots and all that follow, $\phi$ is zero on the positive horizontal axis and increases with counterclockwise rotation, as in the usual two-dimensional case.

techniques such as a one-way ANOVA. Instead, we use a nonparametric test for common mean direction of two sample populations (Fisher, 1993). The test is based on the $Y$ statistic, which plays the role of the $F$ statistic in an ANOVA. Because we do not want to assume a model distribution for the data (i.e., we want a nonparametric test), yet we will be making comparisons between small sample populations, we use a bootstrapping technique to obtain appropriate significance levels. Details of both the $Y$ statistic and the bootstrapping method can be found in the work of Fisher (1993).

\section{RESULTS}

\section{Obstacle rotation}

Participants were able to perform the task easily, colliding with the obstacle or attempting to short-cut behind it on average about six times in a block of 240 successful trials. The mean (SD) movement time across trial blocks was 1143 (314) msec, and the mean (SD) distance from the path near point to the obstacle was $4.2(1.2) \mathrm{cm}$. There were no significant differences in these two measures across obstacle axes or choice of arm used for reaching.

Sample obstacle avoidance paths are shown in Figure 4. The three sets of paths were all taken from the same trial block, but 


\section{Z Axis SG, Right Hand}
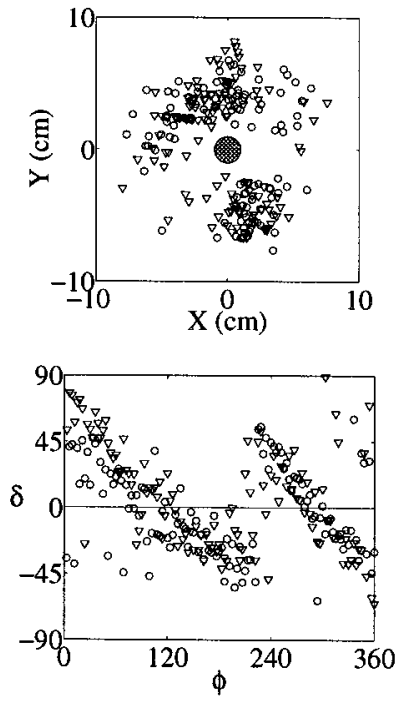

Y Axis CS, Left Hand
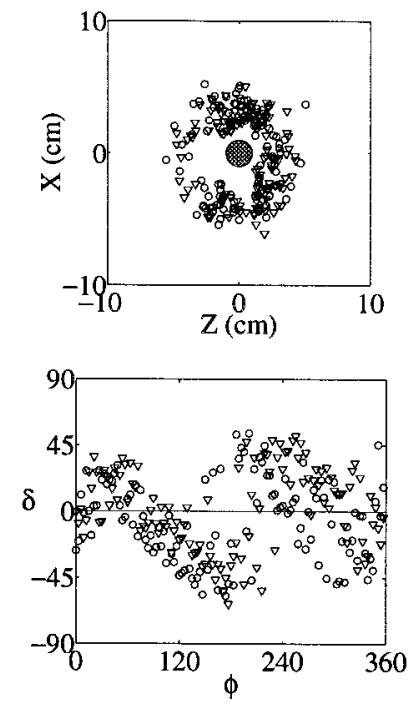

X Axis TF, Right Hand
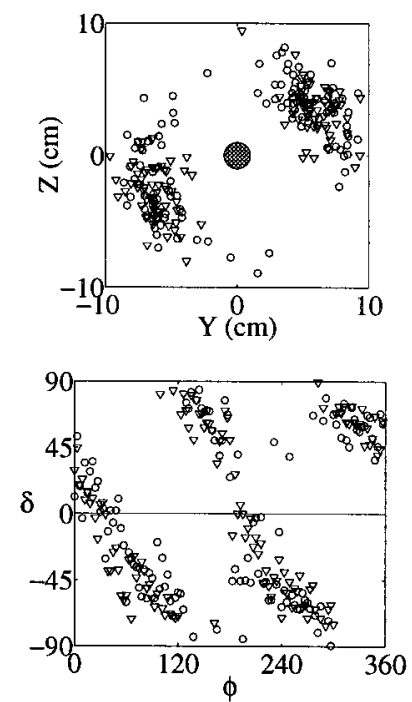

Figure 5. Sample near point results. Top, Near point locations relative to the obstacle in the plane perpendicular to the obstacle length. The obstacle's cross-section is also shown. Bottom, Near point angle versus presentation angle. In all plots, circles are for clockwise movements, and triangles are for counterclockwise movements. the presentation angles are different. There are distinct qualitative differences between the sets of paths. Those in the top row, $\phi$ near $90^{\circ}$, are fairly symmetric, with near points clustering along the line $\delta=0$. When the presentation angle is near $135^{\circ}$, the paths become reliably skewed, and the near points cluster to the right of the presentation axis. Finally, when $\phi$ is near $180^{\circ}$, the obstacle clearance is larger, and the near points cluster at either side of the $\delta=0$ axis. This example illustrates a trend seen for all the participants; near point placement varies as a function of presentation angle in a manner that causes the near points of all paths to cluster toward a preferred axis, in this case approximately aligned with the $X$-axis.

These effects can be seen more clearly by examining all the near points for a particular trial block. Sample near point data for three different participants and three different obstacle orientations are shown in Figure 5. The plots on the top row show a distinct clustering of the near points in the plane perpendicular to the obstacle. Furthermore, the bottom plots, showing near point angle versus presentation angle, are nearly piecewise linear with a negative slope, indicating the existence of a near point preferred axis within each plane.

These preliminary impressions were confirmed with the near point regression analysis. The results are summarized in Figure 6. For every participant, with both hands, and in every plane, the near point regression showed a significant piecewise linear dependence of $\delta$ on $\phi$, i.e., $b$ was significantly $>0$. Furthermore, within an experimental condition, there was no more than approximately a $30^{\circ}$ spread for the preferred axes across participants (with the exception of C.S., $Z$-plane), despite large variations across conditions.

The effect does not seem to be learning-dependent. There is no significant interaction between the strength of the near point clustering (as evidenced by the slope $b$ ) or the quality of fit of the model (as evidenced by the $R^{2}$ statistic) for a trial block and the order of that block. And when the near point regression analysis is performed separately for the first and second halves of each trial block, there is no clear trend in the preferred axis, the strength of the clustering, or the quality of fit of the model.
These results show that three-dimensional, unconstrained obstacle avoidance movements display path variations that are not explicable in terms of the extrinsic task constraints. As is the case with planar, $2 \mathrm{df}$ movements, the near points of the path tend to cluster along a preferred axis that is approximately constant across participants.

\section{Left hand versus right hand}

One explanation for the path variations seen in these experiments is that anisotropies in the perceptual system could lead to different movement plans at different orientations. If the path variability is perceptual in origin, then we would expect it to be the same for both left- and right-hand movements. Alternatively, if the effects are attributable to either the kinematic or dynamic properties of the arm, then we would expect intermanual differences in behavior. In particular, for experiments centered on the midline, as ours were, we would predict that path variations for one arm should be the mirror image of those of the other arm, reflected about the midline.

Figure 7 shows two sets of sample paths from the same participant with the same obstacle orientation and presentation angle, but from movements made with different arms. Note that the paths are skewed in both cases, but the direction of the skew is the opposite in the two cases. Approximately speaking, the obstacle avoidance paths of one arm are the mirror images of the paths of the other, reflected about the line $X=0$.

To make this same comparison over the whole data set, the preferred axes from Figure 6 have been replotted in a circular format in Figure 8. Each annulus corresponds to a single participant, and each pair of arc-shaped boxes marks a preferred axis and $95 \%$ confidence interval. There are two plots for the horizontal $(Z)$ and frontal $(Y)$ planes. The plots on the left display the actual data from the experiment, whereas in the plots on the right, the axes from left-handed blocks are reflected about the midsagittal plane. If movement paths are symmetric about the midline, then data from the two hands should overlap in these latter plots. Paths that lie in the sagittal $(X)$ plane do not change on reflection 

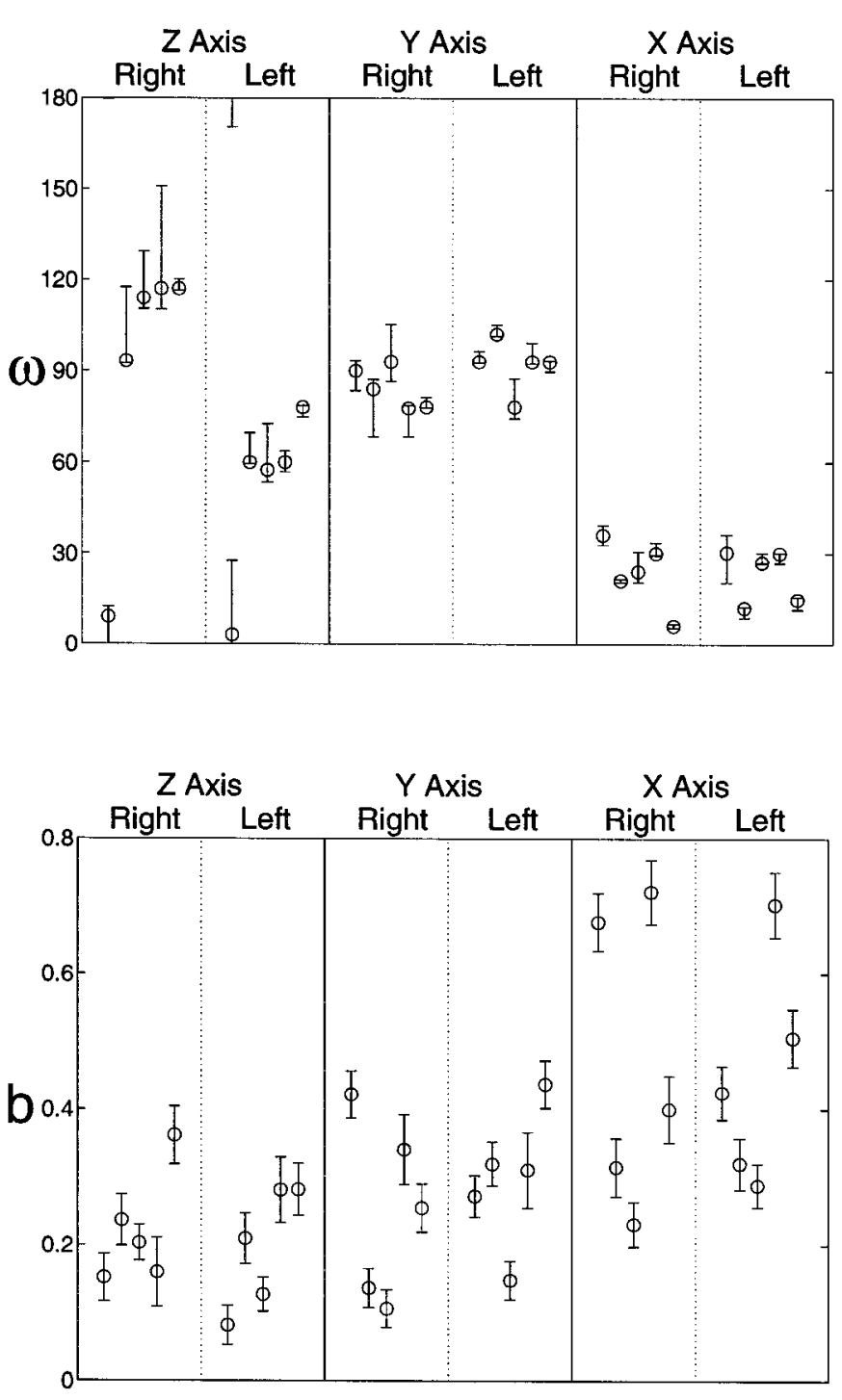

Figure 6. Near point angle regressions. Each point represents the estimated parameter for one participant, and error bars represent $95 \%$ confidence intervals (which are generally not symmetric). Top, Near point preferred axis, $\omega$. Bottom, Regression slope, $b$.

about that plane; therefore, symmetry predicts the same preferred axes for either hand.

For each plane of movement, we performed a nonparametric test for common mean preferred axis between left- and righthand sessions (see Methods, Sensitivity model). For horizontal and frontal plane movements, this analysis was repeated two times, once with the actual data and once with the left-reflected data of Figure 8. The results of the comparisons are shown in Table 1. For $Z$-plane movements, the left- and right-hand preferred axes are significantly different. However, when the lefthand data are reflected about the midline, the two means are statistically indistinguishable. In the case of the $Y$-plane, the preferred axes for both hands lie very near the $X$-axis $\left(90^{\circ}\right)$, so it is difficult to draw any conclusions. However, we note that although there is no significant difference between the groups in either the actual data or the reflected data, the $Y$ statistic is smaller for the reflected data, showing the same trend as that seen for the $Z$-plane. Finally, the $X$-plane data for the two hands have

\section{Right Handed Movements}
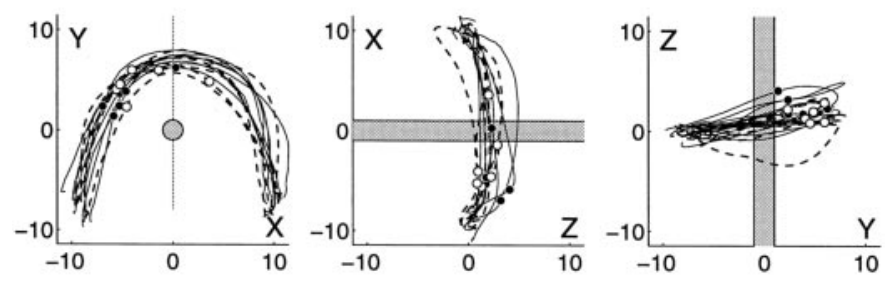

Left Handed Movements
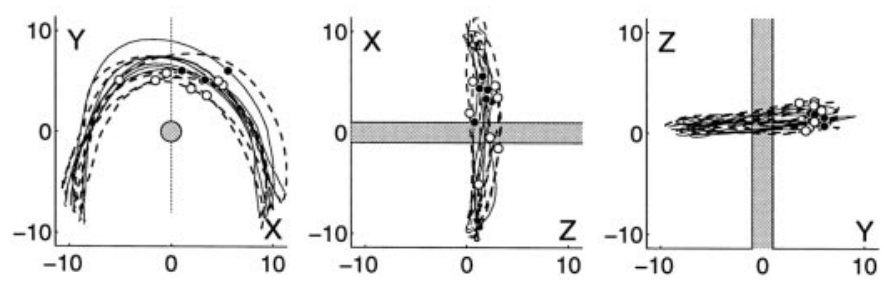

Figure 7. Intermanual path comparisons. Both rows show paths made by T.F. in $Z$-axis trial blocks, but the arm used was different in each case. Each row displays all trials with presentation angles within $10^{\circ}$ of the $Y$-axis. Solid lines are for counterclockwise movements; dashed lines are for clockwise. The black and gray circles mark the counterclockwise and clockwise near points, respectively. The line $\delta=0$ is shown by the dotted line bisecting the obstacle.

nearly identical mean preferred axes, as predicted by symmetry about the midline.

These findings support the claim that the near point placements for movements with the two hands are mirror symmetric about the midline, allowing us to rule out a perceptual origin for the movement asymmetries seen in the obstacle rotation experiment.

\section{Mobility measurements}

To estimate the hand's acceleration we must first specify the width of the temporal window of hand positions to be used in this analysis. There were several factors to consider. First, we wanted to choose as early and narrow a window as possible, because we are trying to estimate the instantaneous acceleration attributable to the perturbative forces. The quality of that estimate will deteriorate as the window becomes too long, for the reasons discussed above (see the discussion on curl in Methods, Mobility measurements). Also, beyond $\sim 100 \mathrm{msec}$, participants can begin to react to the perturbation (Flanders et al., 1986; Flanders and Cordo, 1989). On the other hand the variable errors in the acceleration measurements (and hence the mobility measurements) should decrease as the window gets longer.

Three quantities related to the mobility analysis were considered: the $R^{2}$ statistics for the acceleration and mobility regressions and the curl index. The values of these measures for windows between 20 and $80 \mathrm{msec}$ in duration, beginning at the onset of the force pulse, are shown in Figure 9. The two regressions improved and the curl index decreased as the window size was increased. The plots in Figure 9 suggest that any window beyond $\sim 65 \mathrm{msec}$, at which the curl index first falls to $<0.1$, would be a good choice. We chose a window of $70 \mathrm{msec}$ for the mobility estimates.

Figure 10 compares the mobility minor (stable) axes of the left 


\section{Actual Data}

\section{Left Hand} Reflected About Midline
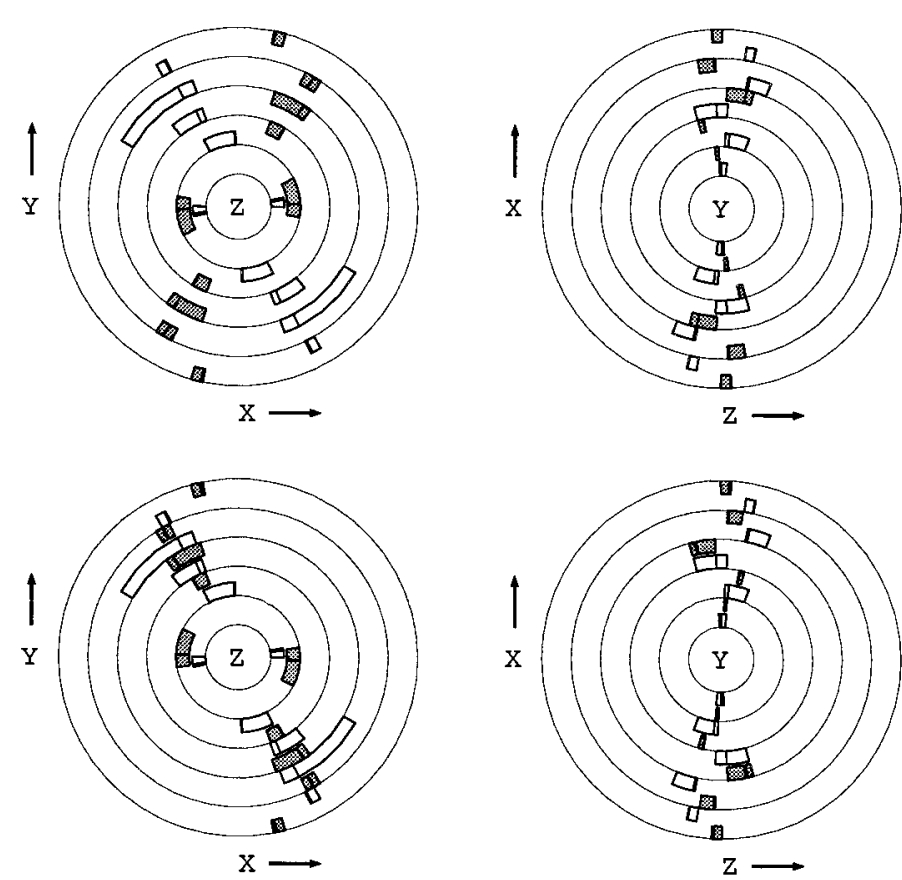

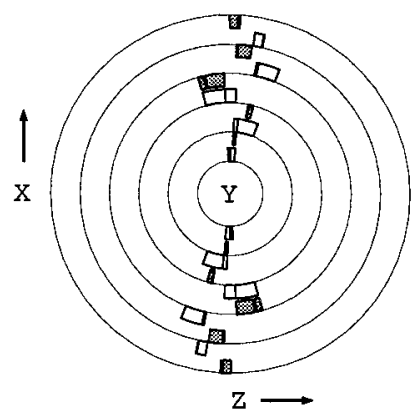

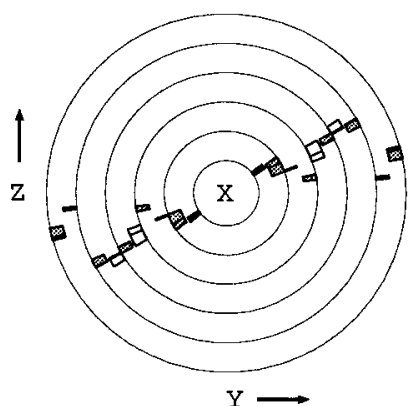

Figure 8. Intermanual comparisons of near point placement. Each plot depicts all the preferred axes for a given obstacle orientation. A single annulus represents one participant and contains two pairs of boxes, each lying along a single axis. The boxes mark the preferred axis (middle line) and 95\% confidence intervals (which are generally not symmetric). The outside gray box represents left-hand movements; the inside white box is for right-hand movements. Top, Actual data. Bottom, Data from the left hand have been reflected about the $X$-axis. The rightmost plot shows the actual results for the $X$-axis movements.

Table 1. Comparisons of mean preferred axis for left- and right-hand sessions

\begin{tabular}{|c|c|c|c|c|c|c|c|c|}
\hline \multirow[b]{2}{*}{ Plane } & \multicolumn{4}{|c|}{ Actual } & \multicolumn{4}{|c|}{ Reflected } \\
\hline & $\bar{\omega}_{\text {right }}$ & $\bar{\omega}_{\text {left }}$ & $Y$ & $p$ & $\bar{\omega}_{\text {right }}$ & $\bar{\omega}_{\text {refl }}$ & $Y$ & $p$ \\
\hline$Z$ & 95.7 & 52.7 & 18.0 & 0.014 & 95.7 & 127.3 & 0.475 & 0.290 \\
\hline$Y$ & 84.5 & 91.8 & 2.70 & 0.156 & 84.5 & 88.2 & 0.657 & 0.423 \\
\hline$X$ & 23.4 & 22.8 & 0.014 & 0.892 & & & & \\
\hline
\end{tabular}

Reflected (refl) refers to data for which the preferred axes in left-hand sessions were reflected about participants' midline. The $Y$ statistic was computed for each comparison, and its probability under the null hypothesis of common mean preferred axis was computed using bootstrapping. Large values for $Y$ indicate a lower likelihood that the two population means are the same (see Methods, Sensitivity model; Fisher, 1993).

and right hands. The results are largely symmetric about the midsagittal plane, as would be expected for the true mobility.

\section{Comparison of mobility measurements and obstacle rotation experiments}

Figure 11 displays each of the measured mobility ellipses, with minor axes drawn in thick lines. Superimposed on those figures are the respective near point preferred axis $95 \%$ confidence regions. Qualitatively, the mobility predictions agree quite well with the observed preferred axes. Only the $Z$-plane data displays a noticeable systematic error, with the near point axes tending more toward the midline than the mobility minor axes.

We assessed the quality of the mobility predictions by using the nonparametric common mean direction test, described in Methods, Sensitivity model. The measured mobility minor axes and the near point preferred axes were compared for each of the six movement plane-hand combinations, and none of the differences approached significance (Table 2). This result could be attributable to small sample size, because there were only four data
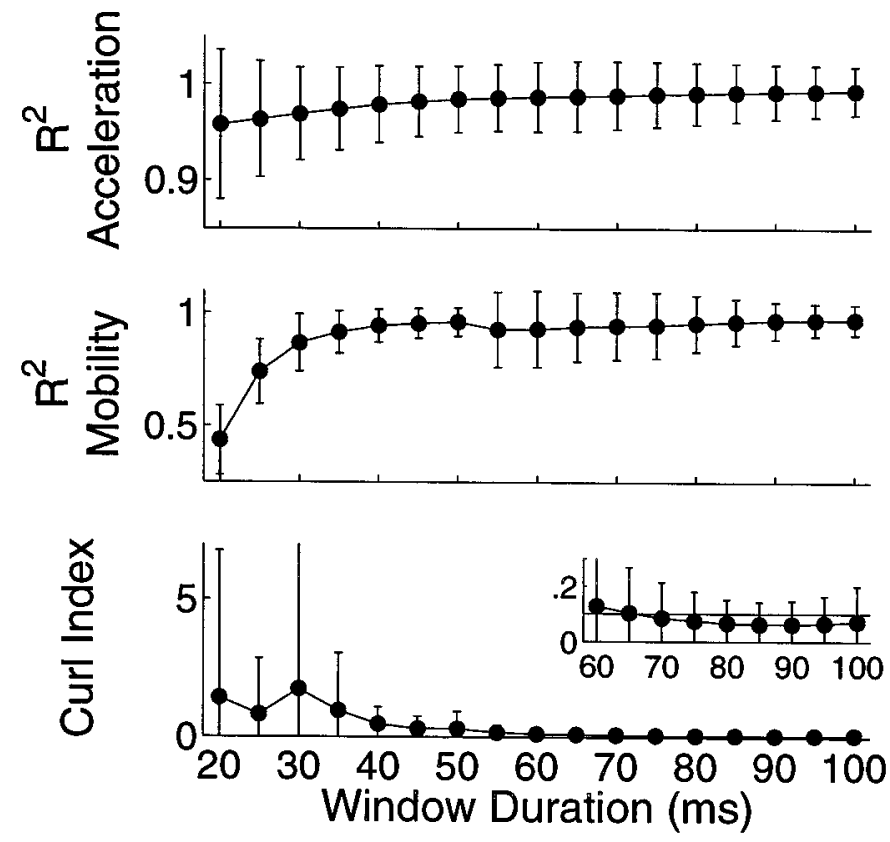

Figure 9. Measures of the quality of the mobility estimate for a range of window sizes. All windows begin at the onset of the force pulse. Each point represents the mean (SD; error bars) across experiments. Top, $R^{2}$ statistic for the acceleration regression. Middle, $R^{2}$ statistic for the mobility regression. Bottom, Curl index of the mobility estimate.

points in each of the groups compared. Thus, for each plane of movement we performed an additional comparison with the data from the left- and right-hand sessions pooled together, after reflecting the left-hand data about the midline. Although the 


\section{Actual Data}

\section{Left Hand} Reflected About Midline
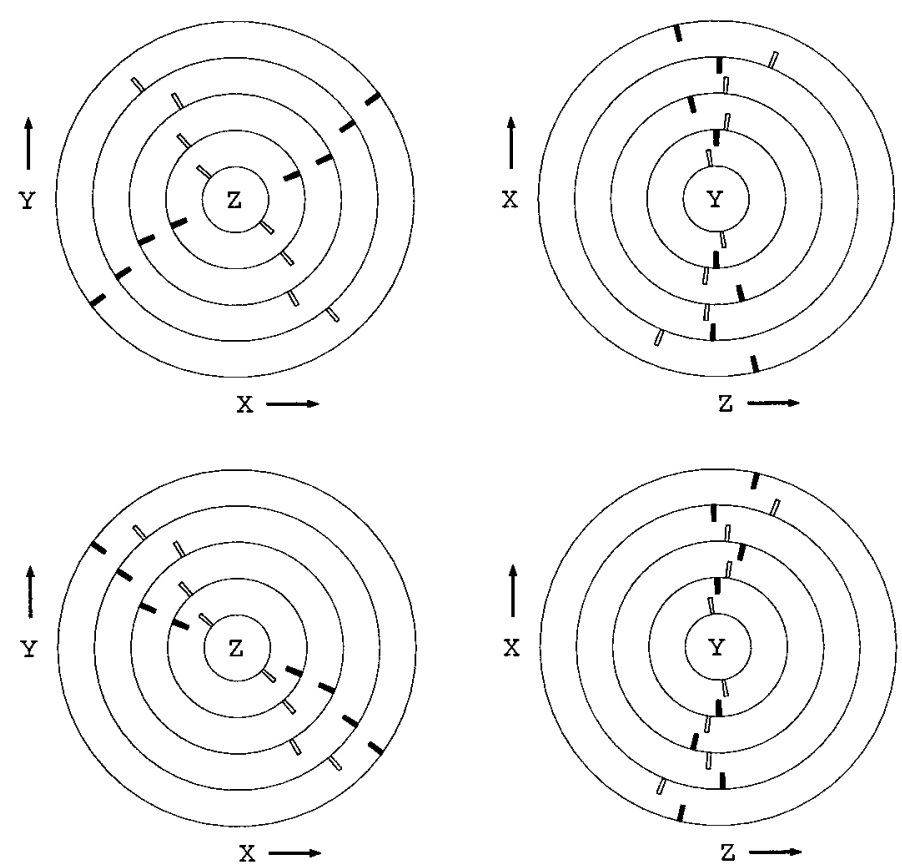

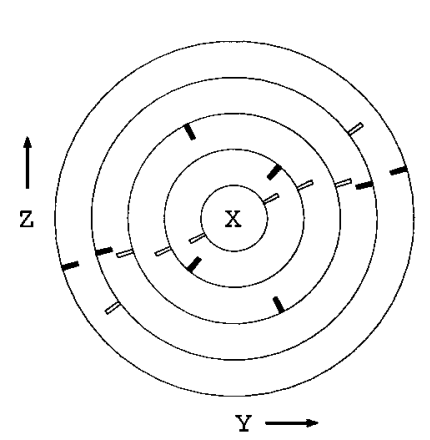

$\mathrm{x}$

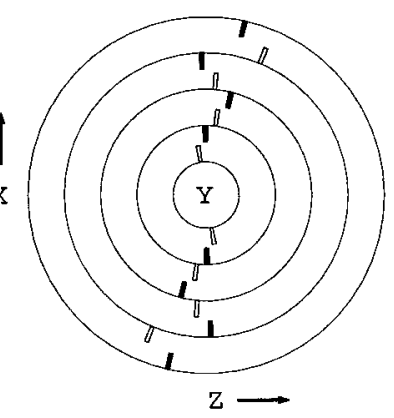

Figure 10. Intermanual comparisons of measured mobility orientations. Each plot depicts all the mobility minor axes for a plane. A single annulus represents one participant and contains two pairs of tick marks each lying along an axis. The outside, black tick marks represent the left hand; the inside, white tick marks are for the right hand. Top, Actual data. Bottom, Data from the left hand have been reflected about the midsagittal plane. The rightmost plot shows the actual results for the $X$-axis movements.

sample sizes were twice as big, still none of the comparisons revealed significant differences.

It should be noted that we did not track participants' posture during the mobility measurements. Differences in posture between the obstacle avoidance and mobility experiments could bias the mobility-based near point axis predictions. However, the two experiments were conducted in same apparatus and with the same visual feedback, and there appeared to be little variation in posture between the two experiments.

\section{DISCUSSION}

There are three main results presented in this paper. First, the three-dimensional, unconstrained obstacle rotation experiment shows predictable variations in movement paths. The near points, where the path makes its closest approach to the obstacle, were not uniformly distributed but tended to cluster on a preferred axis. This distribution was consistent with a previously proposed sensitivity model in which the paths are skewed such that when the hand passes closest to the obstacle, the arm is in a configuration that is most stable to actuator or proprioceptive noise. Second, the near point distribution for the right and left hands showed mirror symmetry about the midline, suggesting that the preferred axis is determined by properties intrinsic to the arm rather than extrinsic factors such as perceptual distortion. Last, measurements of the arm's mobility were made. The minor axis of the mobility matrix, which represents the axis in which the hand is most stable in response to perturbations, was in good agreement with the preferred axis about which the near points of the paths clustered. Together these results provide evidence that knowledge of the stability of the arm is taken into account when planning movements that interact with objects in the environment.

\section{Unconstrained obstacle avoidance}

Obstacle avoidance reaching has been investigated in several previous studies. Abend et al. (1982) asked participants holding the handle of a $2 \mathrm{df}$ manipulandum to reach around a linear obstacle protruding into the straight line path to the target. They found that obstacle avoidance paths displayed high-curvature, low-velocity regions near the tip of the obstacle. This result was modeled by Flash and Hogan (1985), who showed that the minimum jerk trajectory constrained to go through an appropriately chosen via point would display similar kinematics. This work suggests that obstacle avoidance planning can be performed, in large part, by the same types of mechanisms that have already been proposed for unconstrained point-to-point reaching. However, the work does not address the issue of how the via point (or any other appropriate trajectory constraints) would be chosen.

Dean and Brüwer (1994) studied reaching around various lineshaped obstacles at a number of different positions in the workspace. Sabes and Jordan (1997) systematically investigated similar obstacle avoidance movements in a planar version of the experiments presented in this paper. Both of these studies found that obstacle avoidance paths vary over the location and orientation of the movement in the workspace. The latter paper proposed the sensitivity model to account for these variations.

The basic idea behind the sensitivity model is that the planning process takes into account those dynamic characteristics of the arm that affect the difficulty of satisfying the task constraints (e.g., not hitting the obstacle). However, these previous studies comprised movements made while either resting the arm on a table or grasping a planar manipulandum. Recent work comparing pointing movements made on a tabletop with either the unconstrained fingertip or a hand-held cursor found that unconstrained move- 
Z Right
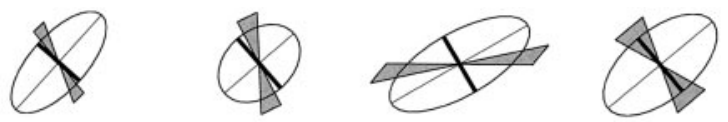

Z Left
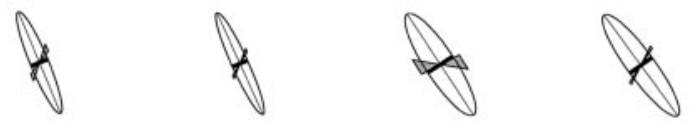

Y Right
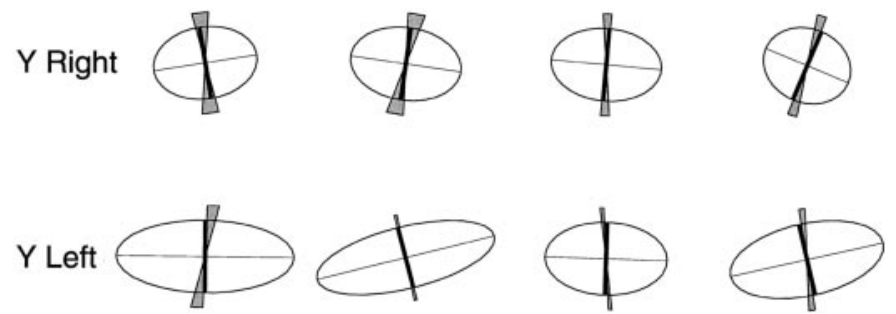

X Right
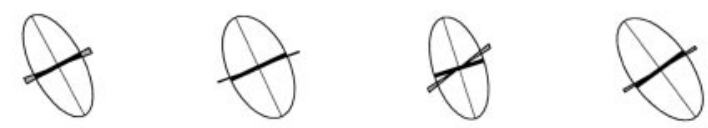

$X$ Left
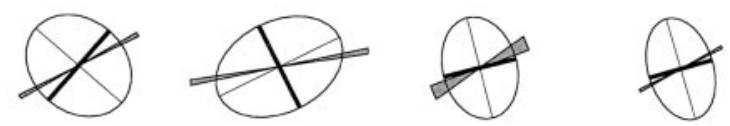

Figure 11. Comparison of measured mobility matrices and obstacle preferred axes. The ellipses represent the estimated mobility matrices, with minor and major axes drawn in bold and thin lines, respectively. The gray wedges show the $95 \%$ confidence regions for the near point preferred axes. The projections onto each plane are the same as those for previous figures, e.g., Figure 5.

ments were more curved that those forced to lie along the tabletop (Desmurget et al., 1997). These results suggest that the nature of interactions with the environment can have a significant effect on movement kinematics. It is thus possible that some of the path variability seen in the planar obstacle avoidance study could be attributable to the interaction between the arm and the table supporting it. The unconstrained movements considered here are free of the artificial constraints inherent in planar movement. We can thus conclude that the anisotropic distribution of near points is attributable to the task constraint under investigation: avoiding a collision with the obstacle.

The task we have examined is a simplified version of real-world obstacle avoidance. Participants were only required to avoid collision with their fingertip, rather than with the entire arm. Similarly, the sensitivity model deals with the sensitivity at the actuator end point (i.e., the fingertip). We chose to study this more tractable task to gain insights into the constraints the CNS uses in motor planning. Although in the physical world additional constraints would likely be required, we believe that the sensitivity criterion captures a significant constraint on the motor planner and that our methods can be extended to explore how other constraints are incorporated into the planning process.

\section{Intrinsic and extrinsic factors}

Although the sensitivity model claims that the observed movement anisotropies are the result of a planning process that ac-
Table 2. Comparisons of mean near point preferred axis and mobility minor axis across participants

\begin{tabular}{crrrl} 
Plane & \multicolumn{5}{c}{$\begin{array}{l}\text { Minor } \\
\text { axis }\end{array}$} & $Y$ & $p$ \\
\hline Right & & & & \\
$Z$ & 29.0 & 49.4 & 0.632 & 0.368 \\
$Y$ & 96.3 & 91.6 & 0.673 & 0.402 \\
$X$ & 25.2 & 24.9 & 0.000 & 0.994 \\
Left & & & & \\
$Z$ & 129.8 & 113.1 & 0.213 & 0.496 \\
$Y$ & 83.4 & 86.2 & 0.183 & 0.591 \\
$X$ & 26.8 & 27.7 & 0.0429 & 0.820 \\
Reflected/pooled & & & & \\
$Z$ & 140.4 & 123.6 & 0.249 & 0.402 \\
$Y$ & 83.6 & 87.3 & 0.745 & 0.358 \\
$X$ & 26.4 & 26.3 & 0.000 & 0.998
\end{tabular}

The mean axes (in degrees) are shown in the first two columns. Comparisons were carried out for each plane with data from the right-hand, the left-hand, and pooled data in which the left-hand data were reflected about the midline. The $Y$ statistic was computed for each comparison, and its probability under the null hypothesis of common mean preferred axis was computed using bootstrapping (see Methods, Sensitivity model; Fisher, 1993)

counts for the arm's dynamics, the data of the earlier planar study could in fact be attributable to purely extrinsic factors. It is known that visual distortions of the workspace can be associated with corresponding distortions in movement path. Wolpert et al. (1994) showed that across participants, path curvature for pointto-point reaching correlates with the perceived curvature of straight lines at the same location in the workspace. Thus, one explanation for the asymmetries seen in the obstacle rotation experiment is that perceptual anisotropies distort the task constraints in a systematic manner as the presentation angle is varied. The planning process in the CNS could then rely exclusively on this distorted visual information for planning movements and still produce paths with the systematic asymmetries observed.

However, any path variability of perceptual origin should look the same independent of the arm used for the movement. In contrast, asymmetries that are based on the kinematic or dynamic characteristics of the arm should exhibit a mirror symmetry about the midline. In fact, we found a mirror symmetry in obstacle avoidance paths. Although there were significant differences between the near point preferred axes with the left and right hands, those differences disappeared when the mirror symmetry was taken into account. This finding rules out a perceptual origin for the path variability in the obstacle rotation experiment and strongly supports the claim that the factors leading to these anisotropies derive from the kinematic or dynamic properties of the arm.

\section{Mobility and the sensitivity model}

Our perturbation-based measurements of the inertia of the arm are similar to previous measurements of the arm's stiffness in the horizontal plane (Mussa-Ivaldi et al., 1985; Gomi and Kawato, 1996) or during single joint movements (Bennett et al., 1992; Bennett, 1993a). The planar stiffness measurements required using data from $300 \mathrm{msec}$ and longer after the force perturbation, raising concerns about whether participants' responses to the perturbation could bias the results. Because our analysis used much smaller time windows $(70 \mathrm{msec})$, we can rule out the effects of central responses, which take on the order of $100 \mathrm{msec}$ (Flanders et al., 1986; Flanders and Cordo, 1989). On the other 
hand, reflex responses to torque perturbations have EMG latencies of $\sim 25 \mathrm{msec}$ (Bennett, 1993b). We argue that these automatic, low-level responses should be considered part of the neuromuscular dynamics, and so including their effects in our measurements is consistent with the spirit of the sensitivity model.

In fact, the predictions of the sensitivity model, based on our empirical estimates of the mobility, showed close agreement with the results of the obstacle avoidance experiment. The preferred axis predictions were statistically indistinguishable from the experimental results.

Our results should be compared with those of Gordon et al. (1994), who investigated point-to-point reaching to targets in a circular array about a fixed origin. They found systematic variations in the kinematics of these movements that are consistent with a movement plan that does not take into account the arm's anisotropic inertia. Because the movement trajectories observed here also vary predictably based on the the inertial properties of the arm, one should consider whether they too might result from a planner and controller that fails to take adequate account of those properties. Such a model would predict very different trajectories for movements made in the clockwise and counterclockwise directions, contrary to what was observed in this study (compare Figs. 4, 7). Furthermore, when participants perform a similar experiment with artificially displaced visual feedback, the preferred near point axis lies closer to that normally observed at the visually perceived location of the arm than that of the arm's actual location (Sabes, 1996).

We argue that the difference between our results and those of Gordon et al. (1994) is attributable to the constraints inherent in the two tasks. In a simple pointing task, there are no external criteria for which the inertial information would make a difference, save the acquisition of the final goal position. And in fact, although the peak acceleration and velocities in the work of Gordon et al. (1994) show a striking match to the inertiaindependent model, the final positional biases show a much weaker effect. Participants were able to vary the movement time to partially offset the inertial effects. The obstacle avoidance task includes a very different kind of constraint, avoiding collision with the obstacle, for which inertial information is useful at a much earlier point in the movement.

Taken together, the experiments of this paper strongly support the notion that the CNS uses intrinsic criteria, based on the arm's dynamics, in the planning of obstacle avoidance movements. These results show that new task constraints lead to new planning strategies, beyond the extrinsic smoothness criteria observable in simple point-to-point movements.

\section{REFERENCES}

Abend W, Bizzi E, Morasso P (1982) Human arm trajectory formation. Brain 105:331-348.

Bennett DJ (1993a) Torques generated at the human elbow joint in response to constant position errors imposed during voluntary movements. Exp Brain Res 95:488-498.

Bennett DJ (1993b) Electromyographic responses to constant position errors imposed during voluntary elbow joint movement in human. Exp Brain Res 95:499-508.

Bennett DJ, Hollerbach JM, Xu Y, Hunter IW (1992) Time-varying stiffness of human elbow joint during cyclic voluntary move. Exp Brain Res 88:433-442.
Bernstein N (1967) The co-ordination and regulation of movements. Oxford: Pergamon.

Dean J, Brüwer M (1994) Control of human arm movements in two dimensions: paths and joint control in avoiding simple linear obstacles. Exp Brain Res 97:497-514.

Desmurget M, Prablanc C, Rossetti Y, Arzi M, Paulignan Y, Urquizar C (1995) Postural and synergic control for three-dimensional movements of reaching and grasping. J Neurophysiol 74:905-910.

Desmurget M, Jordan MI, Prablanc C, Jennerod M (1997) Constrained and unconstrained movements involve different control strategies. J Neurophysiol 77:1644-1650.

Fisher NI (1993) Statistical analysis of circular data. Cambridge, UK: Cambridge UP.

Flanagan JR, Ostry DJ (1990) Trajectories of human multi-joint arm movements: evidence of joint level planning. In: Experimental robotics $\mathrm{I}$, Lecture notes in control and information sciences (Hayward V, Khatib O, eds), pp 594-613. London: Springer.

Flanagan JR, Rao AK (1995) Trajectory adaptation to a nonlinear visuomotor transformation: evidence of motion planning in visually perceived space. J Neurophysiol 74:2174-2178.

Flanders M, Cordo PJ (1989) Kinesthetic and visual control of a bimanual task: specification of direction and amplitude. J Neurosci 9:447-453.

Flanders M, Cordo PJ, Anson JG (1986) Interaction between visually and kinesthetically triggered voluntary responses. J Mot Behav 18:427:448.

Flash T, Gurevich I (1991) Human motor adaptation to external loads. Proc Annu Int Conference IEEE Eng Med Biol Soc 13:885-886.

Flash T, Hogan N (1985) The co-ordination of arm movements: an experimentally confirmed mathematical model. J Neurosci 5:1688-1703.

Gomi H, Kawato M (1996) Equilibrium-point control hypothesis examined by measured arm stiffness during multijoint movement. Science 272:117-119.

Gordon J, Ghilardi MF, Cooper SE, Ghez C (1994) Accuracy of planar reaching movements: II. Systematic extent errors resulting from inertial anisotropy. Exp Brain Res 99:112-130.

Hogan N (1985) The mechanics of multi-joint posture and movement control. Biol Cybern 52:315-331.

Hollerbach JM (1982) Computers, brains, and the control of movement. Trends Neurosci 5:189-192.

Kaminsky T, Gentile AM (1986) Joint control strategies and hand trajectories in multijoint pointing movements. J Mot Behav 18:261-278.

Lackner JR, DiZio P (1994) Rapid adaptation to coriolis force perturbations of arm trajectory. J Neurophysiol 72:299-313.

Morasso P (1981) Spatial control of arm movements. Exp Brain Res 42:223-227.

Mussa-Ivaldi FA, Hogan N, Bizzi E (1985) Neural, mechanical, and geometric factors subserving arm posture. J Neurosci 5:2732-2743.

Sabes PN (1996) The planning of visually guided arm movements: feedback perturbation and obstacle avoidance studies. PhD thesis, Massachusetts Institute of Technology.

Sabes PN, Jordan MI (1997) Obstacle avoidance and a perturbation sensitivity model for motor planning. J Neurosci 17:7119-7128.

Shadmehr R, Mussa-Ivaldi FA (1994) Adaptive representation of dynamics during learning of a motor task. J Neurosci 14:3208-3224.

Soechting JF, Flanders M (1989) Errors in pointing are due to approximations in sensorimotor transformations. J Neurophysiol 62:595-608.

Soechting JF, Lacquaniti F (1981) Invariant characteristics of a pointing movement in man. J Neurosci 1:710-720.

Uno Y, Kawato M, Suzuki R (1989) Formation and control of optimal trajectories in human multijoint arm movements. Minimum torquechange model. Biol Cybern 61: 89-101.

Wolpert DM (1997) Computational approaches to motor control. Trends Cognit Sci 1:209-216.

Wolpert DM, Ghahramani Z, Jordan MI (1994) Perceptual distortion contributes to the curvature of human reaching movements. Exp Brain Res 98:153-156.

Wolpert DM, Ghahramani Z, Jordan MI (1995) Are arm trajectories planned in kinematic or dynamic coordinates? An adaptation study. Exp Brain Res 103:460-470. 